\title{
Fluorescent Probes for Ultrasensitive Cytochemical and Histochemical Imaging
}

\author{
Iain Johnson, Molecular Probes, Inc., 4849 Pitchford Avenue, Eugene, OR, 97405
}

Immunofluorescence techniques using multicolor labeling and analysis of colocalization are essential for unraveling the structural and functional complexity of cells and tissues. Improvements in the capabilities of these techniques are dependent on the development of new dyes and methods for attaching them to antibodies and other markers.

The Alexa Fluor series of dyes have optimized spectroscopic and physical properties that enable preparation of bioconjugates with consistently strong and photostable fluorescence output. The series consists of fourteen dyes with fluorescence excitation peaks ranging from $350 \mathrm{~nm}$ to 750 $\mathrm{nm}$. Functionally important characteristics of Alexa Fluor dyes include strong absorption at the output wavelengths of common excitation sources, resistance to photobleaching and selfquenching, and water solubility to facilitate coupling reactions with proteins and other biomolecules. The well differentiated spectra of the fourteen Alexa Fluor dyes provide many options for multicolor detection (FIG 1) and fluorescence resonance energy transfer (FRET). In addition to their use for immunofluorescent labeling, Alexa Fluor dyes have been incorporated into a wide range of specialized probes including neuronal tracers, fluorescent lipopolysaccharides and cholera toxin B conjugates for detection of lipid rafts.

Simultaneous multicolor immunofluorescence detection requires the use of primary antibodies from unrelated species of animals in conjunction with speciesspecific secondary antibodies, or combinations of biotin-avidin reagents with directly labeled primary and secondary antibodies. The straightforward approach of directly labeling primary antibodies is often overlooked, primarily because chemical coupling of dyes, haptens and enzyme labels to antibodies is a moderately difficult and relatively low-yield process that is impractical for very small quantities of protein. Direct labeling via formation of binary antibody complexes circumvents many of these limitations. Sub-microgram quantities of a primary antibody can be labeled, and the reactions are usually quantitative with respect to the primary antibody. Labeling and purification of the complexes can be completed in a matter of minutes. In this way, customized sets of direct conjugates can be readily prepared for multicolor immunofluorescence applications.

Enzyme-mediated detection methods permit ultrasensitive detection of low abundance targets that are below the detection threshold of other fluorescence techniques. Tyramide Signal Amplification (TSA) and Enzyme-Labeled Fluorescence (ELF) are two complementary techniques that generate signal amplification based on in situ deposition of multiple fluorescent substrates per enzyme label. TSA uses horseradish peroxidase (HRP) enzyme labels and tyramide substrates, which can be coupled to a wide assortment of dyes and haptens. Higher levels of signd amplification can be achieved by detection of Oregon Green 488 tyramide with anti fluorescein/Oregon Green antibody conjugates labeled with HRP, followed by a second round of fluorescent tyramide deposition. The dye-anti-dye primary antibody system also provides a useful alternative to biotin-streptavidin detection in cells and tissues where the presence of endogenous biotin produces substantial background signals. ELF is based on alkaline phosphatase enzyme labels and a fluorogenic substrate that forms a yellow-green-fluorescent precipitate at the site of phosphatase activity. The precipitate is extremely photostable and exhibits an exceptionally large fluorescence Stokes shift, allowing signals to be readily discriminated from background autofluorescence and other fluorescent labels. 
Investigation of physiological responses by fluorescence microscopy requires probes that can be introduced, retained and detected in living cells. Some recent developments in this area include phospholipase substrates used for in vivo imaging of lipid metabolism and fluorescent analogs of phoshointositides that allow the intracellular distributions of these important lipid second messengers to visualized. In addition, new fluorescent indicators for intracellular zinc have been developed that can be used to detect zinc secretion associated with insulin release from pancreatic islets.

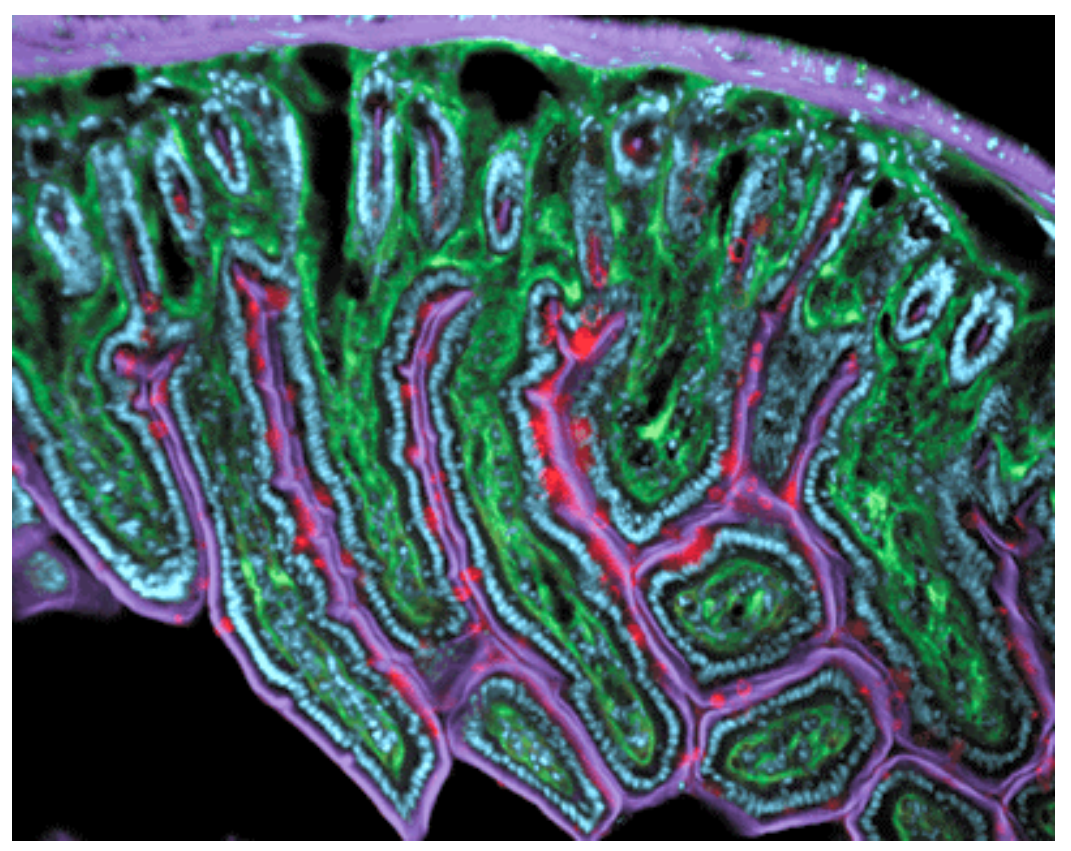

FIG 1. Mouse intestine cryosection showing basement membranes labeled with an antifibronectin antibody and Alexa Fluor 488 goat anti-chicken secondary antibody (green fluorescence). Goblet cells and crypt cells are labeled with Alexa Fluor 594 wheat germ agglutinin (red fluorescence). The microvillar brush border and smooth muscle layer are visualized with Alexa Fluor 680 phalloidin (pseudocolored purple). The section was counterstained with DAPI (blue fluorescence). 